INRA Prod. Anim.,

2012, 25 (2), 113-126

\title{
Le travail des éleveurs avicoles et porcins : une grande diversité de stratégies, d'attentes, de durée et de productivité
}

\author{
G. MARTEL ${ }^{1}$, C. DEPOUDENT 2 , C. ROGUET ${ }^{3}$, S. GALLOT', C. PINEAU 5 \\ ${ }^{1}$ INRA, UR0980 SAD Paysage, 65, rue de Saint-Brieuc, CS 84215, F-35042 Rennes, France \\ 2 Chambre d'Agriculture de Bretagne, Pôle Régional Porc Aviculture, 5 allée Sully, F-29322 Quimper, France \\ ${ }^{3}$ IFIP, Institut du porc, La Motte au Vicomte, BP 35104, F-35651 Le Rheu, France \\ ${ }^{4}$ ITAVI, Service économie, 4 rue de la Bienfaisance, F-75008 Paris, France \\ ${ }_{5}^{5}$ Chambre d'Agriculture de la Sarthe, 15, rue Jean Grémillon, F-72013 Le Mans, France \\ Courriel : gilles.martel@rennes.inra.fr
}

Les études menées dans les filières avicoles et porcines sur le thème du travail montrent, en lien avec les évolutions structurelles du secteur et avec des méthodologies variées, une grande diversité d'attentes, de stratégies et d'efficacité du travail. L'évolution des systèmes de production aura des impacts forts sur le travail des éleveurs et ces impacts doivent donc être pris en compte en amont de ces évolutions.

La durabilité des exploitations d'élevage est au cœur des préoccupations actuelles des politiques européenne et nationale (réforme de la PAC, directive nitrate, conservation des espaces agricoles...), des attentes sociétales (bien être animal, agriculture biologique) et des filières elles-mêmes (labels, image des produits, charte de production...). Ces différents acteurs interrogent les organismes de recherche et de développement sur l'identification, la compréhension et la mise en place effective de systèmes d'élevages durables. Par sa position à l'interface entre l'économie et le social, le thème du travail est d'une importance majeure. En effet, l'augmentation de la productivité des travailleurs (nombre d'animaux produits par travailleur) est un des leviers du coût de production (Reboul 1964), alors que dans le même temps, la charge, l'organisation et le contenu du travail sont des aspects importants pour la qualité de vie du travailleur. Avec un nombre d'animaux à gérer par travailleur plus élevé que dans les autres filières, les élevages de porcs et de volailles sont particulièrement concernés par cette thématique.
Le modèle français d'élevage de porc associe le naissage et l'engraissement : sur les 12700 élevages porcins recensés en 2009, les «Naisseurs-Engraisseurs» (NE) concentrent $85 \%$ des truies du pays et $63 \%$ des porcs à l'engrais. L'étude du travail en production porcine doit tenir compte du fait que l'atelier porcin est, dans $82 \%$ des cas et pour la moitié du cheptel, associé à d'autres productions au sein de l'exploitation, des bovins laitiers pour $34 \%$ des exploitations porcines (et $22 \%$ des porcs), des céréales pour $25 \%$ et des bovins allaitants dans $23 \%$ des cas (tableau 1).

Tableau 1. Typologie des exploitations porcines en 2005 (d'après llari et al 2007).

\begin{tabular}{|l|c|c|c|c|c|c|c|c|c|}
\hline & $\begin{array}{c}\text { Nombre } \\
\text { d'exploitations } \\
\text { en France }\end{array}$ & $\%$ & $\begin{array}{c}\text { Cheptel }_{\text {total }}{ }^{1} \\
\text { en }\end{array}$ & $\%$ & Truies $^{2}$ & $\begin{array}{c}\text { Porcs } \\
\text { engrais }^{2}\end{array}$ & $\begin{array}{c}\text { Total } \\
\text { porcs }^{2}\end{array}$ & $\begin{array}{c}\text { UTA** } \\
\text { totales }^{2}\end{array}$ & $\begin{array}{c}\text { dont } \\
\text { salariées (\%) }\end{array}$ \\
\hline $\begin{array}{l}\text { Spécialisés } \\
\text { porcs }\end{array}$ & 3080 & 18 & 7,64 & 52 & 240 & 1219 & 2481 & 2,9 & 41 \\
\hline $\begin{array}{l}\text { Bovins-lait } \\
\text { et porcs }\end{array}$ & 5710 & 34 & 3,18 & 22 & 40 & 347 & 557 & 2,5 & 12 \\
\hline $\begin{array}{l}\text { Céréales } \\
\text { et porcs }\end{array}$ & 4290 & 25 & 2,25 & 15 & 40 & 314 & 524 & 2,0 & 18 \\
\hline $\begin{array}{l}\text { Bovins-viande } \\
\text { et porcs }\end{array}$ & 3875 & 23 & 1,65 & 11 & 43 & 247 & 425 & 1,7 & 17 \\
\hline Ensemble & 16960 & 100 & 14,63 & 100 & 77 & 494 & 868 & 2,0 & 20 \\
\hline
\end{tabular}

\footnotetext{
1 En millions de têtes.

2 En moyenne par exploitation.
}

** 1 UTA (Unité de Travail Annuel) équivaut au temps de travail d'une personne occupée à temps complet à des travaux agricoles pendant une année. 
Tableau 2. Répartition des exploitations avicoles et surface moyenne des ateliers avicoles en fonction du degré de spécialisation et de la filière de production de l'exploitation.

Données extrapolées : France entière, enquête par sondage détaillé.

Source : élaboration ITAVI d'après AGRESTE - Enquête aviculture 2008.

\begin{tabular}{|c|c|c|c|c|c|c|}
\hline Spécialisation & \multicolumn{2}{|r|}{ Chair } & \multicolumn{2}{|r|}{ Ponte } & \multicolumn{2}{|c|}{ Palmipèdes gras } \\
\hline $\begin{array}{c}\text { (Part du revenu avicole } \\
\text { dans le revenu total } \\
\text { de lexploitation) }\end{array}$ & $\begin{array}{c}\% \\
\text { exploit. }\end{array}$ & $\begin{array}{l}\text { Surface moy. } \\
\text { / exploit }\left(\mathrm{m}^{2}\right)\end{array}$ & $\begin{array}{c}\% \\
\text { exploit. }\end{array}$ & $\begin{array}{c}\text { Surface moy. } \\
\text { / exploit (nb. places) }\end{array}$ & $\begin{array}{c}\% \\
\text { exploit. }\end{array}$ & $\begin{array}{l}\text { Surface moy } \\
\text { / exploit }\left(\mathrm{m}^{2}\right)\end{array}$ \\
\hline $\begin{array}{c}\text { Totale } \\
(\text { Revenu avicole }=100 \%)\end{array}$ & 14,9 & 1569 & 31,4 & 51909 & 22,3 & 4143 \\
\hline $\begin{array}{c}\text { Prépondérante } \\
\text { (Revenu avicole = } \\
75 \text { à moins de } 100 \%)\end{array}$ & 12,5 & 1650 & 19,2 & 35985 & 25,5 & 4959 \\
\hline $\begin{array}{c}\text { Très im portante } \\
\text { (Revenu avicole = } \\
50 \text { à moins de } 75 \% \text { ) }\end{array}$ & 20,4 & 1328 & 20,1 & 23436 & 27,6 & 4466 \\
\hline $\begin{array}{c}\text { Im portante } \\
\text { (Revenu avicole = } \\
25 \text { à moins de 50\%) }\end{array}$ & 33,6 & 981 & 20,9 & 13583 & 15,7 & 3638 \\
\hline $\begin{array}{c}\text { Secondaire/accessoire } \\
\text { (Revenu avicole }= \\
\text { moins de 25\%) }\end{array}$ & 18,6 & 693 & 8,4 & 10281 & 8,9 & 1616 \\
\hline Total & 100,0 & 1169 & 100,0 & 31633 & 100,0 & 4135 \\
\hline
\end{tabular}

En aviculture, le maillon élevage est recentré sur l'engraissement (volailles de chair) ou la production (pondeuses), les étapes de reproduction étant assumées par un maillon distinct (accouvage). Le maillon élevage compte environ 12300 élevages d'engraissement de volailles de chair et 2700 élevages de poules pondeuses destinées à la production d'œufs de consommation (Riffard et Gallot 2010). Les élevages de volailles de chair français se caractérisent par une spécialisation relativement faible de l'atelier avicole sur l'exploitation (tableau 2). La mixité des exploitations avicoles, qui découle pour partie de l'histoire et du modèle de développement de cette production sur les exploitations françaises à partir de la fin des années 1960 (atelier complémentaire, valorisation d'une main-d'œuvre présente...), a eu un impact certain sur la répartition et l'organisation du travail au sein de ces ateliers.

Les systèmes d'élevage porcins et avicoles regroupent les animaux au sein de bâtiments (fermés ou non) et gèrent leur troupeau en lots/bandes. Ce type de conduite est associé au fonctionnement en «tout plein, tout vide» qui consiste à remplir en une seule fois un bâtiment (ou une salle d'un bâtiment) avec des animaux du même stade physiologique (que l'on nomme lot ou bande), à les gérer de manière identique, puis à les en retirer tous ensemble. Entre deux lots, un vide sanitaire est réalisé afin de net- toyer et désinfecter le bâtiment selon un protocole rigoureux. Or, il existe entre élevages et pour l'aviculture, au sein d'un même élevage, une grande diversité de durées de lots et d'intervalles entre deux lots successifs. Ainsi, en élevage avicole, les cycles de production de 4 à 20 semaines alternent avec des périodes de vide sanitaire (Gallot 2008). En élevage porcin, l'intervalle entre deux bandes varie de 1 à 7 semaines selon l'élevage, ce qui influe sur la fréquence à laquelle certaines tâches se répètent (Jégou et al 2010).

Cet article fait le point sur les études récentes menées dans ces deux filières sur les différentes dimensions du travail. Dans un premier temps, nous étudierons l'évolution au cours du temps des structures d'élevage (concentration, agrandissement) et de leur main-d'œuvre (nombre et types de travailleurs). Nous compléterons cette partie pour les porcins par une comparaison européenne de l'efficacité du travail. Nous évoquerons dans la deuxième partie la variabilité des temps et des organisations de travail, et les raisons de cette variabilité. Enfin, nous évoquerons des études récentes dans les deux filières qui prennent en compte l'évolution du métier en abordant notamment les évolutions de la filière, la santé des travailleurs, le contenu du travail des éleveurs, et la diversité de conception de ce métier. Ces thèmes sont de plus en plus importants pour l'attractivité du métier d'éleveur et donc pour la durabilité de ces systèmes d'élevages.

\section{1 / Evolution des structures d'élevages et de la main- d'œuvre}

\section{1 / En production porcine}

a) Concentration et agrandissement des élevages de porcs

Entre 1988 et 2007, plus de 4 exploitations porcines sur 5 ont disparu, essentiellement des petites structures de moins de 100 porcs (Agreste 2008). Dans les exploitations ayant au moins un atelier porcin, la taille de celui-ci augmente avec le temps (figure 1). Ceci est particulièrement vrai pour les ateliers des exploitations «naisseursengraisseurs» qui se sont agrandis de $80 \%$ entre 2005 et 2009 , passant de 99 à 176 truies par élevage. Dans le même temps, en raison d'un contexte économique difficile et de l'obligation de mettre aux normes les locaux pour truies gestantes (conduite en groupe obligatoire à partir du 1er janvier 2013), certains éleveurs de petites structures arrêtent l'atelier naissage de leur exploitation. D'autres font le choix de s'associer pour investir dans des ateliers de naissage collectifs de grande dimension. 
Figure 1. Evolution de la taille des exploitations porcines pratiquant le naissage, par orientation de production.

Source : IFIP d'après enquêtes sur le cheptel du Service de Statistique et Prospective (Agreste).

Entre 2005 et 2009, la taille a été multipliée par 1,8 pour les naisseurs $30 \mathrm{~kg}$ et 1,1 pour les Naisseurs-Engraisseurs (NE).

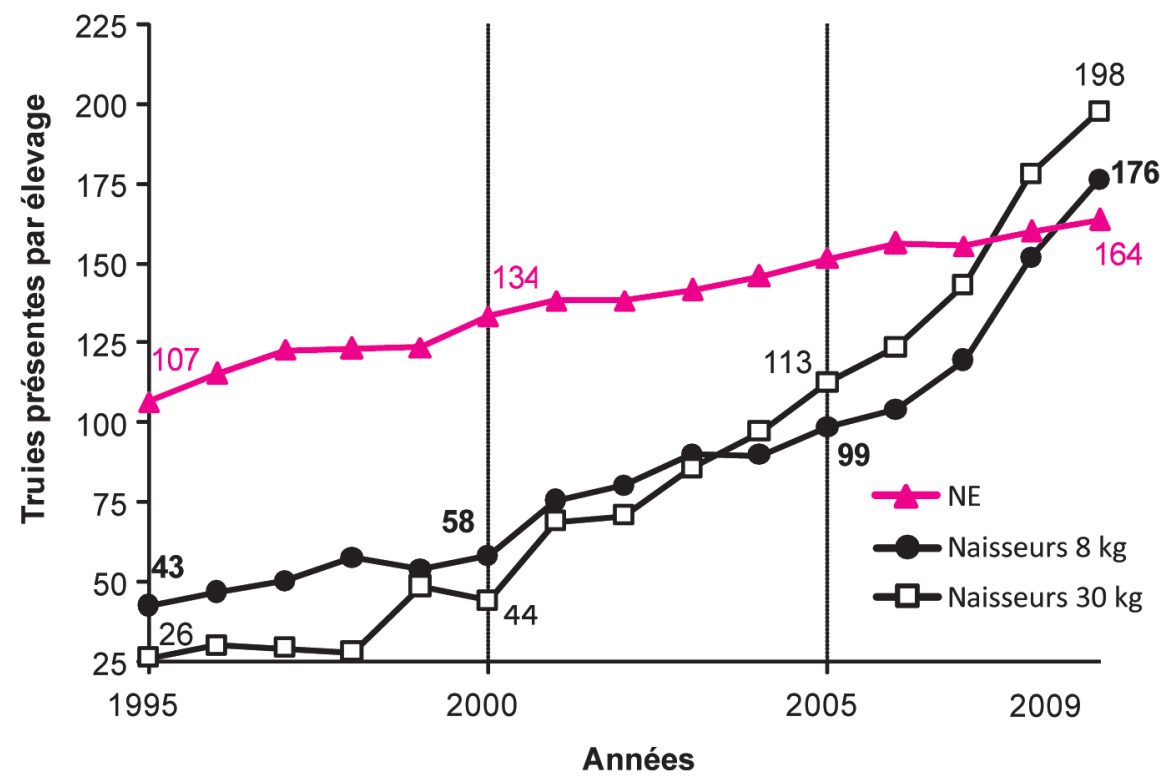

b) Augmentation de la part de maind'œuvre salariée

Le nombre de travailleurs (exprimé en UTA par exploitation ${ }^{1}$ ) a fortement diminué au cours des dernières années, passant de 151500 en 1995 à 60000 en 2007. Cette diminution est principalement due à l'arrêt d'exploitations non spécialisées et à l'amélioration de la productivité du travail. En effet, les exploitations porcines spécialisées emploient un effectif à peu près stable de 8000 UTA alors que leur cheptel a cru de 5,1 à 6,8 millions de porcs : la productivité apparente du travail a augmenté de 731 à 850 porcins/UTA dans les exploitations porcines spécialisées sur la période.

Parallèlement, le recours au salariat s'est développé. En 2007, 39\% de la main-d'œuvre dans les exploitations porcines spécialisées était salariée, contre $24 \%$ en 1995. Cette moyenne recouvre des situations variées. Ainsi, près de $70 \%$ des exploitations porcines n'emploient aucun salarié (les moins spécialisées, avec les ateliers porcins les ainsi $141 \mathrm{~kg}$ de carcasse soit le niveau atteint par les danois et par le tiers des meilleurs élevages français classés sur le temps par truie. En revanche, en moyenne, un éleveur français est au niveau de l'éleveur allemand avec 103 $\mathrm{kg}$ de carcasse (Roguet et al 2011). Les différences d'efficacité sur le naissage traditionnel sont édifiantes : 3,3 porcelets produits par heure de travail aux Pays-Bas, 2,6 au Danemark mais seulement 1,6 en France (Roguet et al 2011).

A l'échelle de l'exploitation, les données de l'enquête du RICA ${ }^{4}$ sur les comptabilités des agriculteurs dans les états membres de l'UE confirment cette hiérarchie. En 2008, un travailleur dans une exploitation porcine spécialisée, pratiquant le naissage et l'engraissement, gère 169 truies au Danemark et 156 aux Pays-Bas contre 94 en France et 88 en Allemagne. En naissage spécialisé (avec post-sevrage), le nombre de truies gérées par travailleur est de 226 au Danemark et 229 aux Pays-Bas, contre 134 en Allemagne. Ceci est d'autant plus impressionnant pour le Danemark que la place des cultures est aussi importante. Le recours au salariat est massif au Danemark $(65 \%$ des UTA en NE, $75 \%$ en naissage spécialisé) et peu important en Allemagne (17\% en NE, $28 \%$ en naissage, $16 \%$ en engraissement), les autres pays étant dans une situation intermédiaire (39\% en France et 30\% aux Pays-Bas en NE) en 2008. Les meilleurs élevages français atteignent les niveaux d'efficacité du travail danois et néerlandais, témoignant que l'écart entre pays se creuse surtout avec le retard de modernisation et d'investissement en France. En effet, la concentration drastique des élevages dans le nord de l'Europe - disparition de 7 élevages de truies sur 10 en 10 ans - s'accompagne d'investissements conséquents dans la modernisation et la rationalisation des bâtiments d'élevages (Roguet et Rieu 2011). En témoigne le capital d'exploitation par truie (hors foncier, quota et autres droits) supérieur au Danemark et aux Pays-Bas. Avec l'agrandissement des ateliers, se généralisent les équipements facilitant le travail (caillebotis, automatisation), l'emploi salarié, la spécialisation des tâches et la recherche d'économies d'échelle (conduite

\footnotetext{
${ }^{1}$ L'Unité de Travail Annuel (UTA) équivaut au travail d'une personne travaillant à temps plein pendant une année.

2 Interpig est un réseau d'experts en économie de la production porcine issus d'une douzaine de pays d'Europe et du continent américain. Il calcule les coûts de revient du porc dans chaque pays participant à partir des données de performances techniques et des prix des facteurs de production, à dires d'experts ou d'après les dispositifs de suivi, entrés dans un modèle de calcul standardisé.

3 Truie en production : de la première saillie à la réforme $=$ truie présente / 1,096

4 Créé en 1965 pour suivre les effets de la Politique Agricole Commune (PAC), le Réseau d'Information Comptable Agricole (RICA) repose sur une enquête réalisée à partir des comptabilités des agriculteurs dans les états membres de l'UE selon "des règles et des principes communs». Il permet la constatation annuelle des revenus agricoles et l'analyse du fonctionnement économique des exploitations. Le RICA couvre «l'ensemble des exploitations agricoles professionnelles», au sens statistique, c'est-à-dire d'une dimension économique supérieure à un seuil de marge brute standard propre à chaque pays et employant au moins 0,75 UTA.
} 
Tableau 3. Comparaison européenne de la productivité du travail en élevage porcin Naisseurs-Engraisseurs (NE). Source : IFIP d'après Interpig et GTE-Tableau de bord.

\begin{tabular}{|c|c|c|c|c|c|c|}
\hline & & Allemagne & France & \begin{tabular}{|c|} 
France \\
Tiers \\
supérieur
\end{tabular} & $\begin{array}{l}\text { Pays- } \\
\text { Bas }\end{array}$ & Danemark \\
\hline \multirow{6}{*}{$\begin{array}{l}\text { Interpig } \\
\text { année } \\
2009\end{array}$} & $\begin{array}{l}\text { Taille moyenne des élevages de } \\
\text { l'échantillon (en truies) }\end{array}$ & 175 & 187 & 196 & 348 & 579 \\
\hline & $\begin{array}{l}\text { Temps de travail (h/truie/an) } \\
\text { hors FAF" et hors gestion } \\
\text { déjections }\end{array}$ & 19,7 & 21,3 & 13,9 & 16,6 & 15,1 \\
\hline & Kg carcasse produits/truies/an & 2084 & 2189 & 1963 & 2349 & 2064 \\
\hline & Coût du travail $(€ / h)$ & 15,2 & 17,5 & & 20,7 & 21,5 \\
\hline & Kg carcasse produits/h & 106 & 102 & 146 & 141 & 137 \\
\hline & Nombre de porcelets produits/h & 1,78 & 1,59 & & 3,25 & 2,55 \\
\hline \multirow{6}{*}{$\begin{array}{l}\text { RICA } \\
\text { année } \\
2008\end{array}$} & \begin{tabular}{|l|} 
Taille moyenne des élevages \\
de l'échantillon (en truies)
\end{tabular} & 169 & 225 & & 304 & 566 \\
\hline & Surface Agricole Utile (ha) & 56,4 & 41,2 & & 12,5 & 145,0 \\
\hline & UTA & 1,91 & 2,38 & & 1,95 & 3,35 \\
\hline & dont salariée & $17 \%$ & $39 \%$ & & $30 \%$ & $65 \%$ \\
\hline & Truies/UTA & 88 & 94 & & 156 & 169 \\
\hline & Capital d'exploitation/truie $(€)$ & 2268 & 2607 & & 3507 & 3650 \\
\hline
\end{tabular}

* FAF : Fabrication d'Aliments à la Ferme.

** UTA (Unité de Travail Annuel) équivaut au temps de travail d'une personne occupée à temps complet à des travaux agricoles pendant une année.

en grandes bandes, gestion). Enfin, dans le nord de l'Europe, le poids du travail dans le coût de revient (15 à $20 \%$ pour un porcelet) conduit les professionnels et les financeurs à porter une attention croissante à ce facteur de production coûteux. Une heure de travail coûte en effet $23 \%$ plus cher au Danemark qu'en France.
Dans les élevages danois et néerlandais, la meilleure efficacité du travail ne s'accompagne pas d'un meilleur revenu de l'éleveur (pour rémunérer son travail familial et ses capitaux propres). Elle repose en effet sur d'importants investissements, financés largement par l'emprunt, dont les intérêts payés consomment une grande partie du résultat
(Roguet 2011, tableau 4). Mais accroître l'efficacité du travail permet aussi d'améliorer les conditions de sa réalisation, notamment via l'automatisation et de réduire la dépendance à ce facteur de production parfois rare, le travail en élevage porcin jouissant d'une faible attractivité.

Tableau 4. De la production agricole au résultat courant des exploitations porcines naisseurs-engraisseurs dans 4 pays de I'UE. Source : IFIP d'après RICA.

\begin{tabular}{|c|c|c|c|c|}
\hline Moyenne en k€ sur 2004-2008 & Pays-Bas & Danemark & France & Allemagne \\
\hline Production agricole totale & 673,5 & 869,7 & 463,8 & 281,1 \\
\hline consommations intermédiaires & $-510,0$ & $-610,6$ & $-361,2$ & $-212,2$ \\
\hline (=) Valeur Ajoutée Brute (VAB) & 163,5 & 259,1 & 102,7 & 68,9 \\
\hline Solde subvention d'exploitation et taxes & 0,7 & 39,5 & 12,7 & 19,7 \\
\hline $\begin{array}{l}\text { Solde subvention et taxes sur } \\
\text { investissement }\end{array}$ & $-0,7$ & 0,35 & 1,3 & $-2,6$ \\
\hline Salaires & $-16,5$ & $-87,7$ & $-17,9$ & $-6,5$ \\
\hline Fermages & $-10,3$ & $-34,6$ & $-6,4$ & $-11,4$ \\
\hline (=) Excédent Brut d'Exploitation (EBE) & 136,6 & 176,7 & 92,3 & 68,2 \\
\hline Amortissements & $-50,9$ & $-101,9$ & $-45,9$ & $-29,6$ \\
\hline Intérêts payés & $-38,0$ & $-130,0$ & $-14,7$ & $-7,8$ \\
\hline (=) Revenu Courant Avant Impôt (RCAI) & 47,7 & $-55,1$ & 31,6 & 30,7 \\
\hline \multicolumn{5}{|l|}{ Rémunération du travail } \\
\hline VAB/UTA ${ }^{1}$ & 88,4 & 71,5 & 45,4 & 37,6 \\
\hline EBE/UTA Familial & 99,8 & 146,9 & 61,0 & 44,7 \\
\hline RCAI/UTA Familial & 34,8 & $-45,8$ & 20,9 & 20,1 \\
\hline
\end{tabular}

1 Une UTA (Unité de Travail Annuel) équivaut au temps de travail d'une personne occupée à temps complet à des travaux agricoles pendant une année. 


\section{2 / En production avicole}

a) Une concentration modérée et toujours une faible spécialisation des exploitations

En production avicole, l'accroissement des capacités de production (évaluées via la surface des bâtiments d'élevage avicole) n'a pas été aussi rapide que dans les élevages porcins. Entre le début des années 1980 et la fin des années 1990, la filière volailles de chair a répondu à l'accroissement de la demande en augmentant le nombre d'éleveurs et d'ateliers d'une part, et la productivité des élevages d'autre part. Les ateliers ainsi créés sont venus diversifier l'activité d'exploitations existantes. La capacité moyenne des élevages a donc modérément augmenté, de l'ordre de $3 \%$ par an, pour s'établir fin 2008 à un peu moins de $1200 \mathrm{~m}^{2}$ de bâtiments d'élevage par atelier. Cet historique explique en partie le fait qu'aujourd'hui encore, seulement $30 \%$ des exploitations ayant un atelier avicole tirent plus de $75 \%$ de leurs revenus de cet atelier.

Dans le même temps, le fort développement du nombre d'exploitations en productions alternatives, estimé en 2008 à près de 4900 exploitations en volailles de chair (dont plus de 4300 en Label Rouge) et 1100 exploitations en pondeuses (dont près de 850 en plein-air et Label Rouge), aux capacités de production souvent limitées par les cahiers des charges, a aussi contribué à limiter l'évolution de la capacité moyenne des ateliers et la concentration des élevages.

L'impact sur la main-d'œuvre a été en général bien ajusté, car souvent l'atelier avicole a été créé parce qu'il existait une disponibilité de main-d'œuvre, immédiate comme dans le cas d'un salarié pas totalement occupé, ou en perspective proche, à travers un projet d'installation d'un enfant sur l'exploitation familiale ou du retour sur l'exploitation de l'épouse travaillant à l'extérieur par exemple (Riffard et Gallot 2010).

A partir du début des années 2000, la filière est entrée dans une période de crise structurelle de surproduction. Dans ce contexte de crise, deux stratégies s'observent et coexistent : la stratégie de «la branche coupée», qui consiste à se séparer de l'activité sinistrée et la stratégie de la «survie à tout prix», plus courante chez des structures spécialisées, qui consiste à tenter d'accroître les capacités afin d'améliorer encore la productivité et la compétitivité de l'activité. La principale réponse observée sur cette période a plutôt été l'option «branche coupée» (chute de $21 \%$ du nombre d'exploitations productrices de volailles de chair entre 2004 et 2008, Agreste 2008) pour deux raisons principales : la faible capacité d'investissement dégagée sur cette période, où les marges ont sensiblement baissé, et la non spécialisation des exploitations avicoles, qui a permis d'abandonner l'atelier avicole pour recentrer sur d'autres activités, souvent dominantes, de l'exploitation. Ces cessations d'activité avicole sont présentes dans toutes les régions mais le Grand Ouest (Bretagne, Pays-de-laLoire, Poitou-Charentes), déjà principale zone de production, résiste mieux (- $15,6 \%$ en moyenne pour ces trois régions). Cette forte diminution $\mathrm{du}$ nombre d'exploitations de volailles n'a engendré qu'une baisse limitée de la production de volailles de chair $(-7,5 \%$, Agreste 2008), non pas via l'agrandissement des ateliers avicoles mais grâce à une augmentation du nombre de cycles de production par atelier. Au final, cette crise a donc eu peu d'effet sur la taille des ateliers avicoles.

La filière ponte n'a pas traversé les mêmes difficultés et le secteur semble s'être concentré assez rapidement : la capacité moyenne par exploitation est passée d'un peu plus de 18800 poules en 2004 à près de 25500 poules en 2008 (Gallot 2006, Riffard et Gallot 2010). Cette concentration rapide s'observe à la fois pour les élevages en cages et pour les élevages alternatifs. Mais aux dires des acteurs du terrain elle ne semble pas avoir bouleversé l'organisation du travail dans les exploitations, sans doute en raison du fort degré d'automatisation des tâches les plus chronophages (alimentation et ramassage des œufs).

b) Une main-d'œuvre principalement familiale, un recours régulier à des travailleurs extérieurs

En l'absence d'évolutions majeures de la taille des ateliers, la structure de la main-d'œuvre a également peu évolué. Elle reste très centrée sur une maind'œuvre familiale, qui assure les tâches courantes de surveillance et de soins aux animaux, avec un recours régulier à des travailleurs extérieurs pour des tâches plus ponctuelles (nettoyage/désinfection des bâtiments entre les lots, mise en place des animaux et surtout enlèvement). Le cycle de production de volailles de chair alterne de longues périodes à besoin de personnel modéré avec de brèves périodes à très fort besoin de main-d'œuvre (souvent autour d'une dizaine de personnes). Cette forte variabilité du besoin de travailleurs tout au long du cycle, qui se répète entre 3 et 7 fois par an (selon les productions) est une contrainte forte, qui rend la seule main-d'œuvre de l'exploitation (familiale ou salariée) insuffi- sante à certaines étapes clés et oblige presque systématiquement au recours à des travailleurs extérieurs, dont l'origine peut être diverse (embauches ponctuelles, sous-traitance à des sociétés de prestation, recours au bénévolat...). Dans un tel contexte, le développement $\mathrm{du}$ salariat reste peu important. L'externalisation des tâches les plus lourdes, par le recours à des sociétés de prestation de services, a en revanche plutôt tendance à se développer depuis quelques années. Mais même externalisées, ces tâches nécessitent que les chefs d'exploitation prennent en compte les conditions et la pénibilité du travail. En effet, ces tâches souvent ingrates et difficiles à automatiser ne doivent pas nuire à l'attractivité du métier, et donc à la capacité à trouver du personnel lorsqu'ils en ont besoin. Le ramassage mécanisé des animaux, développé depuis la fin des années 1990, réduit nettement le besoin en personnel et fait partie d'initiatives visant à trouver des alternatives dans l'organisation de ces étapes critiques sur le plan de la maind'œuvre. Mais d'autres contraintes (sanitaires, configuration des bâtiments, organisation des chantiers...) ont fait que le ramassage mécanisé est loin d'être généralisé aujourd'hui.

\section{3 / Rôle de la segmentation sur l'évolution des filières porcine et avicole}

Une des raisons de la différence d'évolution dans les structures d'élevage des deux filières est la segmentation de leur marché respectif. En production porcine, la part des élevages sous signe officiel de qualité est minime par rapport à la production totale nationale. De plus, dans les labels de qualité de cette filière ce sont les modes de transformation et d'affinage de la viande qui sont ciblés plus que la façon dont a été élevé le porc charcutier, laissant les éleveurs libres de déterminer la taille de leur structure. En revanche, historiquement en volailles de chair, la production label rouge contribue à une forte segmentation du marché de la volaille de chair. Or, le cahier des charges de ce label induit une capacité maximale de production (1600 $\mathrm{m}^{2}$ par site d'exploitation) et se traduit donc par des tailles de structures qui ne peuvent pas être dépassées. Depuis quelques années, on assiste à ce phénomène en poules pondeuses avec une hausse de la production pour des ateliers ayant un parcours, ou permettant la ponte au sol, au détriment des poules élevées en cages. En effet, depuis l'apparition de l'affichage du type d'élevage des poules sur la coquille des œufs, le consommateur s'est tourné plus largement vers l'œuf coquille «poules en plein air», «Label rouge» et «bio» (qui représentent aujourd'hui respecti- 
vement 16,8 et $8 \%$ des volumes achetés) et délaisse peu à peu l'œuf coquille standard (qui représente néanmoins $67 \%$ des volumes mais seulement $53 \%$ de la valeur). Ces nouvelles productions sont exigeantes en temps de travail mais bénéficient d'une bonne image et sont rémunératrices, ce qui a séduit de nouveaux agriculteurs, issus ou non du monde agricole.

\section{2 / Organisation et temps de travail : variabilité et facteurs de variation}

Dès les années 1970, des études se sont intéressées au travail à l'échelle d'une exploitation en se focalisant particulièrement sur le temps de travail et l'organisation des tâches entre les différents ateliers (Daelemans 1972, Bassez 1973, ITP et IGER 1973, 1976a, 1976b). Cette entrée était motivée par l'hypothèse que «l'amélioration de la productivité du travail conditionne d'une façon croissante la rentabilité des entreprises» (Reboul 1964). Concernant l'organisation du travail en élevage porcin, les études se sont d'abord focalisées, en raison du nombre d'animaux important à gérer par exploitation, sur la mise en œuvre de la conduite en bandes. En effet, cette conduite a été réfléchie pour optimiser l'utilisation des bâtiments, produire des lots importants d'animaux prêts à être abattus en même temps et répartir la charge de travail au cours du temps (Caugant 2002). Les études plus récentes regardent l'organisation du travail à des échelles temporelles plus fines (semaines/week-ends) (Martel et al 2008) ou en prenant en compte l'ensemble des ateliers de l'exploitation, grâce à l'adaptation de la méthode du Bilan Travail (Grannec 2010a). L'organisation du travail se réfléchit un peu différemment en élevage avicole. En effet, un élevage de volailles de chair peut, en fonction des demandes du marché, être amené à élever différentes espèces (poulets de chair et dindes principalement). Il peut les produire en alternance ou, s'il possède plusieurs bâtiments, de manière simultanée. La durée des cycles de production des différents types d'animaux n'est pas la même, ce qui peut engendrer des problèmes d'organisation à certaines périodes. Une autre différence entre l'organisation du travail en élevage porcin et en élevage de volailles de chair réside dans la capacité de ce dernier à s'ajuster au rythme des autres ateliers de part leur courte durée. Ainsi, une fois un lot de volailles de chair achevé, un éleveur dispose en général d'une certaine marge de manœuvre (sous réserve d'une anticipation suffisante) pour décaler la mise en place du lot suivant de quelques jours pour tenir compte du calendrier des cultures. Ceci permet de caler un (ou plusieurs) lots entre deux pics de travail sur l'exploitation. L'évaluation du temps de travail, pour sa part, est généralement faite à partir d'une partition précise des tâches selon leur périodicité : tâches quotidiennes (alimentation, soins aux animaux, paillage et curage), tâches périodiques liées à la succession des lots (nettoyage/désinfection, transferts d'animaux...), tâches définies à l'échelle de l'élevage et pouvant être sporadiques ou au contraire très réparties (enlèvement des lisiers et épandage, gestion administrative et économique, entretien des bâtiments...). En production porcine, ces évaluations suivent une partition des tâches décrites dans des grilles élaborées par l'IFIP il y a près de 30 ans et intégrées dans une application de gestion dite "Gestion Technico-économique - Tableau de bord». Elles ont permis de constituer un observatoire permanent sur le temps de travail en élevage, de produire des références pour les différentes catégories d'élevages (naisseurs, naisseurs-engraisseurs, engraisseurs), d'évaluer les progrès réalisés au cours du temps, de situer les élevages individuellement au sein d'une population et en conséquence, de fixer, les concernant, des priorités d'amélioration. Ces données sont régulièrement complétées par des études ponctuelles réalisées par les Chambres d'Agriculture ou 1'IFIP (Salaün 1987 et 1992, Le Borgne 1992, Le Borgne et al 1994, Le Borgne et Quentric 2002, Le Moan et al 2003, Bartolomeu 2005, Renaud 2010). En production avicole, le temps de travail fait uniquement l'objet d'études ponctuelles. Ces études se sont focalisées sur les temps de productions en volailles de chair (Guerder 2001) et en volailles reproductrices (Chevalier et Guerder 2004), en poules pondeuses en agriculture biologique (Pineau et Morinière 2009). Les objectifs de ces études étaient d'obtenir des références sur les temps de production et leur cinétique, et d'identifier des facteurs permettant d'optimiser le temps de travail. Ces facteurs pouvaient être matériels (bâtiment, équipement), structurels (spécialisation, dimension de l'atelier) ou humain (main-d'œuvre, formation).

\section{1 / En production porcine}

a) Des rythmes très liés à la conduite en bandes

Dans les élevages de porcs «naisseurs-engraisseurs», le principal facteur jouant sur l'organisation du travail est le nombre de bandes dans le troupeau. En effet, du choix de ce critère découle la succession des tâches à réaliser dans l'exploitation (tableau 5, Caugant 2002). Le mode de conduite en bandes est déterminé par des considérations techniques (optimisation de l'occupation des bâtiments, recherche de performances), mais aussi par la volonté d'améliorer les conditions de travail (Caugant 2002). Ainsi, Le Moan et al (2009) notaient que de nombreux éleveurs changeaient de conduite pour dégager du temps libre, améliorer la productivité du travail, ou réduire la

Tableau 5. Enchaînement des actes techniques périodiques dans 3 conduites en bandes en élevage porcin.

\begin{tabular}{|c|c|c|c|}
\hline Semaine & 20/21 bandes & 7 bandes & S bandes \\
\hline 1 & $\begin{array}{c}\text { Sevrage, détection des } \\
\text { œstrus, IA, mises bas }\end{array}$ & Sevrage \\
\hline 2 & $\begin{array}{c}\text { Sevrage, détection des } \\
\text { œstrus, IA, mises bas }\end{array}$ & Détection des œstrus, IA & $\begin{array}{c}\text { Détection des œstrus, IA, } \\
\text { Mises bas }\end{array}$ \\
\hline 3 & $\begin{array}{c}\text { Sevrage, détection des } \\
\text { œestrus, IA, mises bas }\end{array}$ & Mises bas & - \\
\hline 4 & $\begin{array}{c}\text { Sevrage, détection des } \\
œ \text { strus, IA, mises bas }\end{array}$ & Sevrage & - \\
\hline
\end{tabular}

IA : Insémination Artificielle. 
Figure 2. Evolution du temps de travail par truie et du nombre de truies par UTA dans les élevages naisseurs-engraisseurs français participants au suivi GTETableau de bord de I'IFIP.

Source : IFIP GTE-Tableau de Bord, élevages naisseurs-engraisseurs.

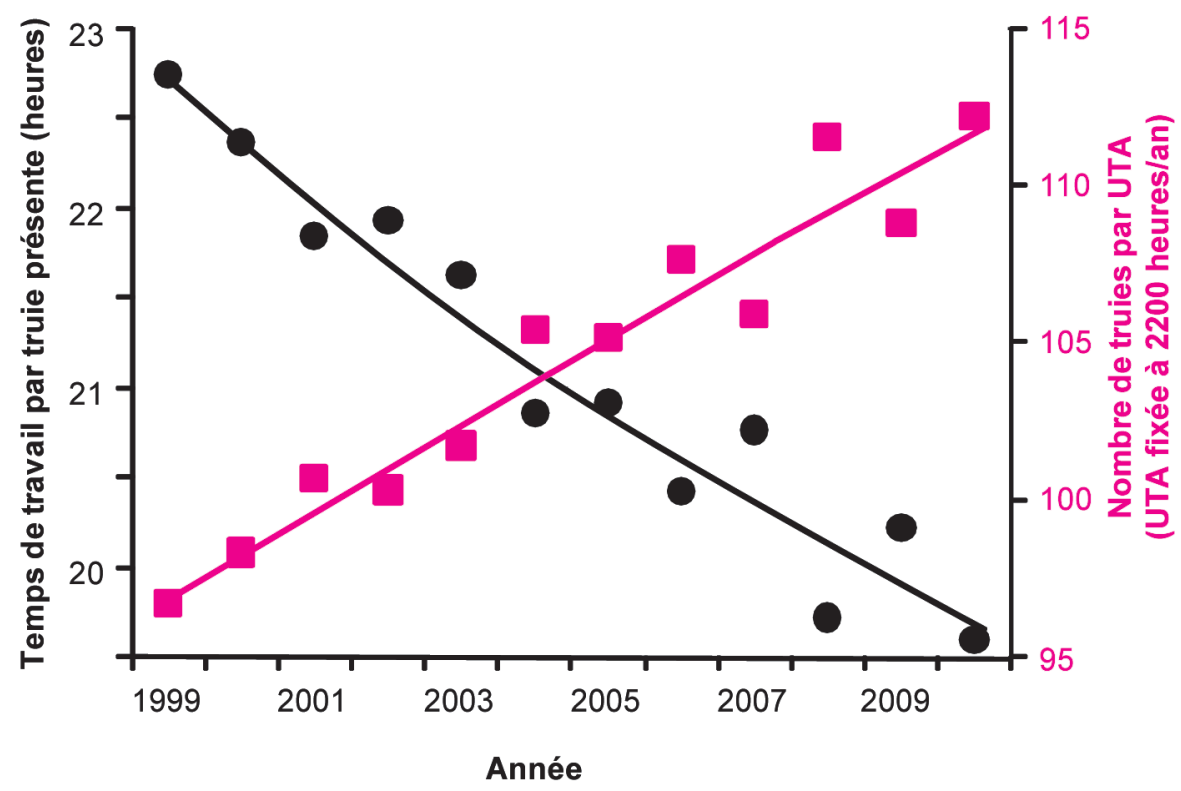

routine liée à des conduites très régulières. La mise en place d'une conduite en cinq bandes permet par exemple de décharger deux semaines sur quatre, mais elle engendre des pics de travail lors d'une semaine (avec insémination et mises bas) (Martel et al 2008, Grannec 2010a). La conduite en sept bandes permet de répartir le travail entre les semaines et la conduite en vingt bandes (souvent dite «conduite à la semaine») permet de gérer de grands effectifs et de spécialiser des travailleurs sur les différentes tâches.

b) Des temps de travaux qui diminuent mais qui restent très variables

Le temps de travail dans un élevage naisseurs-engraisseurs, ramené à la truie présente, a diminué de $11 \%$ entre 1999 et 2009 , passant en moyenne de 22,7 à 20,2 heures par truie présente et par an (figure 2). En moyenne, un travailleur à temps complet gère environ 110 truies dans ces élevages. Le temps de travail sur l'atelier porcin, en dehors de la gestion et de la fabrication d'aliments à la ferme, est majoritairement consacré au naissage (entre 59 et $65 \%$ du temps dédié à cette activité selon les études) (Le Moan et al 2003, Renaud 2010, Roguet et al 2011). La tâche la plus chronophage est l'alimentation et la surveillance quotidienne des animaux $(46 \%$ du temps, Coefficient de Variation $^{5}=53 \%$ ), devant les soins et la surveillance en maternité $(17 \%, \mathrm{CV}=$
$62 \%)$, le nettoyage-désinfection (12\%, $\mathrm{CV}=56 \%)$ et les transferts $(9 \%, \mathrm{CV}=$ $43 \%$ ) (Renaud 2010). Toutes les études montrent une variabilité très importante des résultats pour chacun des postes étudiés. Outre l'imprécision d'une telle mesure, la variabilité du temps de travail s'explique par des différences entre élevages au niveau de leurs équipements (mécanisation, organisation des bâtiments...), de leurs tailles mais aussi de leurs pratiques (Le Moan et al 2003, Martel et al 2008, Roguet et al 2011). Par exemple, la mise en place d'un système de prétrempage automatique permet de diminuer le temps associé à ce travail peu apprécié des éleveurs (Corrégé et Theil 2008). Sur l'effet taille, Le Moan et al (2003) montrent qu'en moyenne, les éleveurs avec des troupeaux de plus de 400 truies passent $30 \%$ moins de temps par truie que les éleveurs de troupeaux de moins de 150 truies.

c) Des pratiques de reproduction liées aux attentes de production et d'organisation du travail

Martel et al (2008) ont mis en évidence le rôle des attentes des éleveurs dans leurs pratiques de gestion des animaux. Ainsi, les attentes productives orientent le choix des pratiques de gestion des mises bas et des nouveaux nés alors que les attentes de rythme de travail influencent les pratiques de gestion des œestrus et des inséminations. Plus précisément, ces auteurs remarquent une distinction entre des éleveurs qui surveillent et aident les truies et les porcelets lors de la mise bas (fouilles, hormones, biberons, adoptions...) et ceux qui laissent faire les animaux. Les premiers ont des objectifs de nombre de porcelets par truie plus élevés que les seconds. Les éleveurs établissent généralement une priorité entre la détection des œstrus et les inséminations : ceux qui font plusieurs détections par jour font généralement moins d'inséminations par truie et inversement. Les éleveurs adaptent aussi leurs protocoles d'insémination soit selon l'intervalle sevrage-œstrus (un protocole par jour de la semaine), soit selon la parité des truies (un protocole pour les cochettes, un pour les primipares, un pour les truies). Le choix d'un protocole est lié aux attentes de l'éleveur vis-à-vis de l'organisation du travail au cours de la semaine et notamment sa répartition entre la semaine et le week-end. Enfin, cette diversité de stratégies se retrouve dans toutes les conduites en bandes : les attentes d'organisation du travail à l'échelle de la semaine et les attentes autour de la mise bas sont indépendantes des attentes d'organisation du travail sur plusieurs semaines.

d) Impact de la taille du collectif de travail sur l'organisation

Dans les exploitations où plusieurs personnes travaillent sur l'atelier porcin, la gestion des rythmes de travail est légèrement différente de celle des exploitations individuelles. Les possibilités d'entraide réduisent la charge individuelle lors des pointes de travail. Ainsi, l'étude de grands collectifs de travail avec des équipes stables montre l'existence de systèmes d'entraide institutionnalisés : par binômes (ex : personnel de verraterie et personnel de maternité) ou selon les disponibilités après concertation lors de la pause matinale (Depoudent et Le Moan 2011). Cette taille de collectif permet également d'ajuster les horaires aux besoins de l'atelier (transferts, naissances, lavage) mais aussi des personnes qui y travaillent. En revanche, les périodes de congés peuvent être source de tensions, sauf dans les élevages ayant recours à des remplaçants ou fonctionnant avec un léger sureffectif (Depoudent et Le Moan 2010). Les horaires de travail sont toutefois jugés très positivement par les salariés, qui apprécient leur régularité et les jugent facilement conciliables avec la vie de famille (Depoudent et Grannec 2011).

\footnotetext{
5 Le Coefficient de Variation (CV), correspond à l'écart-type de l'échantillon divisé par sa moyenne, exprimé en pour cent.
} 


\section{2 / En élevage de volailles de chair et de pondeuses}

a) Variabilité des temps de travaux selon les espèces

Les deux principales études sur les temps de production concluent à une forte variabilité des temps de travaux. Les temps de travail sont très variables entre espèces en volailles reproductrices (Chevalier et Guerder 2004) : le coefficient de variation varie entre $48 \%$ (élevage de canes de Barbarie reproductrices) et $60 \%$ (dindes reproductrices). Cette variabilité découle en partie de tâches spécifiques ou plus chronophages d'une espèce à l'autre. Les temps de «travaux ponctuels» et de «nettoyage» montrent de fortes variations. Par exemple, la nécessité de pailler les nids en élevage de canes, les différences de mode de reproduction, les interventions sanitaires spécifiques sont autant de facteurs qui expliquent un écart du simple au double entre le temps le plus faible (poule) et le plus élevé (dinde).

La variabilité des temps est également prononcée, pour une espèce donnée, au sein de tâches bien identifiées : elle est particulièrement forte pour la tâche de mise en place des abreuvoirs et des mangeoires en élevage de dindes reproductrices (CV de 128\%).

b) Temps de travaux à l'échelle des exploitations

Une étude plus récente (Gallot 2011) a eu pour objectif de transposer aux élevages de monogastriques la méthode Bilan Travail, mise au point au début des années 1990 pour les exploitations de ruminants. L'étude, qui a impliqué une cinquantaine d'élevages avicoles et porcins et environ 550 exploitations de ruminants, a permis de collecter et d'analyser de manière homogène la structure du travail sur des exploitations et des ateliers très différents. L'objectif prioritaire de cette étude était de tester la transposition de la méthode aux élevages de monogastriques, sur un échantillon le plus varié possible. En parallèle, l'étude a permis de produire les références globales pour ces productions (voir Cournut et Chauvat (2012) dans ce numéro).

La méthode Bilan Travail distingue deux types de travaux : le «Travail d'Astreinte» (TA), quotidien et non différable dans le temps, et le «Travail de Saison» (TS), qui intervient plus ponctuellement et qui peut, dans une certaine mesure, être différé. En aviculture, les tâches de surveillance et de soins quotidiens aux animaux ont été classées en travail d'astreinte et les interventions ponctuelles en début et fin de lot (prépa-
Figure 3. Relation entre le Travail d'astreinte (TA) des ateliers avicoles et la taille des ateliers (d'après Gallot 2011).

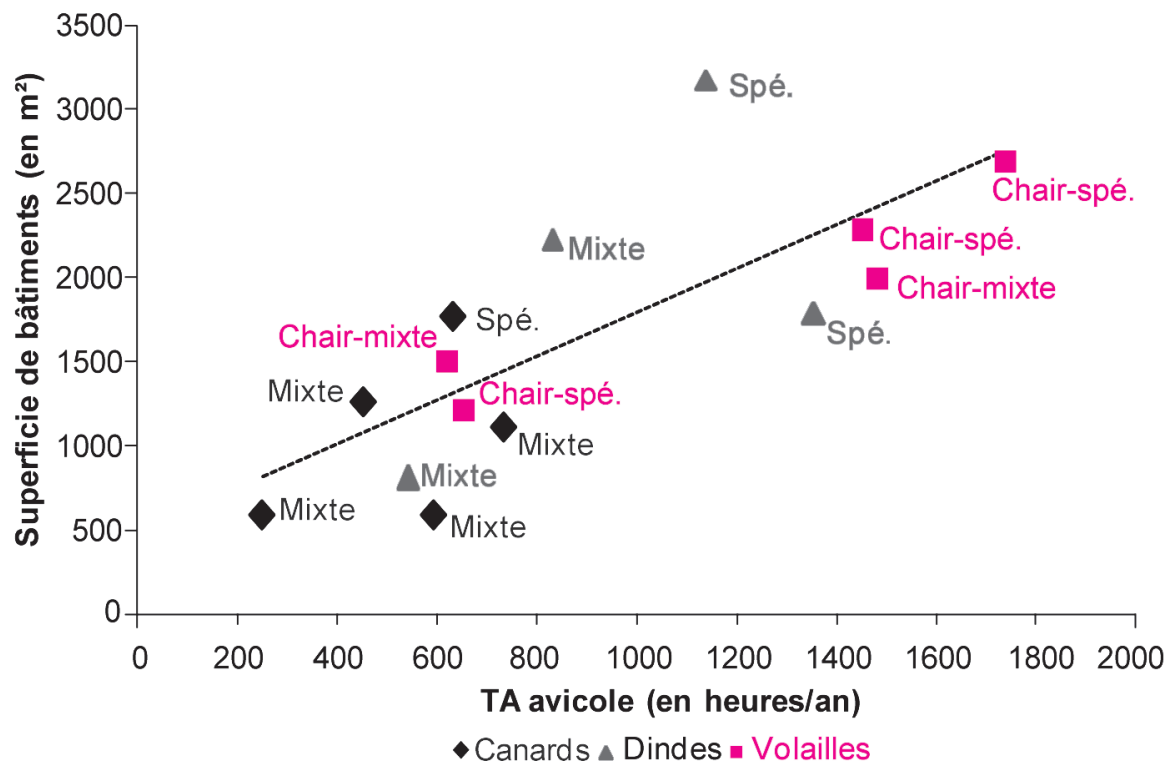

ration du bâtiment, mise en place des animaux, enlèvement, nettoyage et désinfection) ont été qualifiées de travail de saison. Cette approche a été mise en œuvre et éprouvée sur 24 exploitations avicoles très diversifiées (en termes de production, dimension...) par les Chambres d'Agriculture de Bretagne et des Pays de la Loire et par l'ITAVI. Elle a permis de mesurer que le TA moyen s'établit à un peu plus de 1700 heures par an pour l'atelier avicole, mais varie presque du simple au triple selon les filières : autour de 1100 heures pour la filière «volailles de chair» et environ 3000 heures pour la filière «volailles de ponte». Ces valeurs moyennes dépendent bien entendu de la capacité des élevages, mais aussi de la spécialisation des exploitations. Ainsi, le travail d'astreinte avicole des exploitations mixtes est presque toujours inférieur de moitié au travail d'astreinte avicole des exploitations spécialisées. La représentation du travail d'astreinte avicole en fonction de la capacité des ateliers (figure 3) amène à plusieurs constats. Tout d'abord, il existe une relation positive entre la taille de l'élevage avicole et le travail d'astreinte total consacré à l'élevage avicole. Cette relation semble être linéaire, ce qui indiquerait qu'il n'existe pas d'économie d'échelle sur le TA. Mais, plus l'atelier grandit, plus la variabilité du TA augmente : pour des structures de capacités et de productions similaires (par exemple pour des ateliers de canard à rôtir de $600 \mathrm{~m}^{2}$ ), le TA peut varier du simple $(200 \mathrm{~h} / \mathrm{an})$ au triple (600 h/an). Enfin, quand les éleveurs mixtes ont des capacités identiques aux éleveurs spécialisés, ils paraîssent consacrer moins de TA à leur atelier avicole $\left(0,57\right.$ heure $/ \mathrm{m}^{2} / \mathrm{an}$ contre
0,64 heure $/ \mathrm{m}^{2} /$ an en moyenne). Meilleure productivité du travail chez ces éleveurs mixtes ou moindre implication dans l'atelier ? La mise en relation de ces données sur le travail avec les résultats technico-économiques apporterait une dimension supplémentaire à l'analyse. L'étude confirme également que l'essentiel du temps de travail $(86 \%$ du travail d'astreinte avicole et $55 \%$ du travail de saison avicole) est assuré par la cellule de base (surtout de la maind'œuvre familiale), le reste du travail étant assuré par du salariat, du bénévolat ou par un recours fréquent à des entreprises de prestation (enlèvement des animaux, nettoyage des bâtiments...).

Dans une approche plus globale de l'exploitation, l'analyse de l'ensemble des Bilans Travail tend à montrer que les productions de volailles de chair présentent des forts points communs avec viande, de même que les élevages de pondeuses se rapprochent des exploitations laitières. Les contrastes sont en revanche forts entre filières avicole et porcine, notamment entre volailles de chair et porcs (voir Cournut et Chauvat (2012) dans ce numéro). Ainsi, le rapprochement entre les productions porcines et avicoles peut sembler cohérent sur le plan des modes d'élevage (élevage en lots, dans des bâtiments plus ou moins fermés) et de la conduite des animaux (cycle court, animaux monogastriques, alimentation sans fourrage, distribution d'un aliment complet acheté ou fabriqué à la ferme...), mais il s'avère peu pertinent dès lors que l'on traite des questions d'organisation et de temps de travail. les exploitations à dominante de bovins 
c) Les facteurs de variation : un rôle prépondérant de l'éleveur

Les auteurs de ces études ont tenté de mettre en relation la variabilité des temps de travaux avec trois facteurs susceptibles d'influer sur cette variabilité : le type de bâtiment, le type d'équipement et l'éleveur. Mais il a été difficile de mettre clairement en relation les temps de travaux avec ces critères. Pour les élevages reproducteurs, les auteurs ont testé certaines dépendances : temps de préparation et de nettoyage en fonction du type de sol (bétonné ou en terre battue); temps de préparation et temps de travaux quotidiens selon le type de ramassage (automatique, semi-automatique ou manuel); temps de travaux quotidiens selon la position des nids (en position centrale ou disposés parmi les animaux); temps de travaux quotidiens et ponctuels avec la pratique du paillage des nids, temps de travaux quotidiens et ponctuels avec la pratique de l'insémination artificielle; temps à l'arrivée des animaux et temps d'enlèvement selon le mode de transport des animaux (containers ou cages). En général les corrélations sont faibles ou inexistantes. Les auteurs insistent sur l'importance du facteur éleveur, qui est sans doute la principale source de variabilité, tant en élevage de volailles reproductrices qu'en engraissement. Dans un même bâtiment, avec les mêmes équipements et pour une même production, deux éleveurs pourront passer des temps très différents en fonction de critères personnels multiples (attrait personnel pour la production, motivation, disponibilité en temps par rapport aux autres ateliers...), qui dépassent la seule compétence. Ces critères interviennent aussi sur le temps que l'éleveur consacre à son atelier. Dans ces études, la relation entre le temps consacré à l'atelier et le niveau des résultats technico-économiques n'a pas été recherchée.

\section{3 / Des métiers diversifiés : de la gestion administrative à la gestion de la santé des travailleurs}

\section{1 / Conceptions de métiers dif- férentes}

L'étude des facteurs de variation des pratiques, des formes d'organisation du travail et des temps de travaux ne doit pas faire oublier la diversité intrinsèque liée à des conceptions du métier d'éleveur différentes. Ainsi l'étude des «styles d'élevage», réalisée par Commandeur et al (2006) auprès d'éleveurs porcins des Côtes d'Armor, met en évidence cinq styles, correspondant à des priorités différentes dans la gestion du travail. Les «entrepreneurs intensité» ou «entrepreneur échelle», gérant de gros élevages avec plus d'un salarié, s'axent sur la division du travail et le recours à une main-d'œuvre non familiale. Les «artisans», passionnés par les performances techniques, privilégient la maîtrise technique à la libération de temps libre. Les «animaliers», passionnés par les animaux et notamment le travail en maternité, peuvent être freinés par des bâtiments peu efficaces. Quant aux éleveurs de type «patrimoniaux», ils inscrivent leur travail dans une tradition familiale et mobilisent leur entourage proche. En termes de relations avec les animaux, les éleveurs porcins se positionnent plutôt comme des éleveurs «avec l'animal», voire «pour l'animal» dans les élevages de reproducteurs (Dockès et Kling-Eveillard 2007). Autrement dit, les éleveurs «avec l'animal» apprécient d'effectuer un travail technique avec des animaux mais ils ne s'attachent toutefois pas individuellement aux animaux.

Pour les salariés en élevage porcin, population croissante parmi les travailleurs de cette production, les motivations sont assez similaires à celles des éleveurs. Ainsi, deux enquêtes réalisées auprès de salariés bretons (Depoudent et Le Moan 2010, Depoudent et Grannec 2011) montrent que le choix de ce métier résulte d'une combinaison entre un marché du travail accueillant, l'envie de travailler avec des animaux (pour certains spécifiquement avec des porcs) et le désir de travailler selon des horaires de type «de bureau». Dans la durée, les aspects concrets du métier, l'autonomie accordée par l'employeur et les relations avec les animaux sont identifiés comme des facteurs de satisfaction et de motivation (Depoudent et al 2012).

\section{2 / Evolution du contenu du travail par l'automatisation et la gestion administrative}

Dans les années 1980 et 1990, l'automatisation croissante des élevages et l'augmentation des quantités d'informations disponibles sur les animaux (suivi de carrière, identification électronique avec distribution automatique de concentré...) ont modifié les travaux réalisés en élevage, les tâches physiquement exigeantes se voyant remplacées par des travaux de surveillance et de gestion de l'information (Nicourt et Souron 1989). La charge physique a été réduite, tandis que les cadences et les besoins de compétences croissaient. Avec la mise aux normes des truies gestantes (obligatoire à partir de 2013), l'organisation du travail change à nouveau. Les éleveurs doivent trouver de nouvelles manières d'observer leurs animaux, notamment dans les élevages où de grands troupeaux sont conduits avec un Distributeur Automatique de Concentré (DAC). Cette évolution semble ne causer ni plus ni moins de travail (Ramonet et al 2010). Que ce soit en production avicole ou production porcine, la maîtrise des outils informatiques et technologiques devient toutefois de plus en plus déterminante. C'est aussi le cas pour les bâtiments qui deviennent de plus en plus complexes notamment avec l'arrivée des systèmes de ventilation assistée par des ventilateurs extracteurs d'air. Le pilotage du bâtiment, de son ambiance et le suivi des animaux nécessitent donc une forte technicité de la part de l'éleveur. Des tâches comme le ramassage des volailles, assuré à la main par l'éleveur et une équipe de journaliers d'une dizaine de personnes entre 2 à 6 fois par an selon la production, ont aussi trouvé des voies d'automatisation. Les machines de ramassage, qui se sont développées depuis la fin des années 1990, ont permis de réduire à la fois la pénibilité du travail (ramassage réalisé en général en fin de nuit, dans la poussière...) et le stress qui pèse sur l'éleveur par rapport à l'organisation du chantier de ramassage (équipes de journaliers parfois difficiles à constituer, peu formées, personnes se désistant quelques heures avant le ramassage, management d'une équipe...). En 2010, on estimait que moins d'un tiers des poulets (et très peu de dindes) étaient ramassés par des machines. Néanmoins l'avancée est suffisamment significative pour être signalée. Enfin, la mise en place, dans les filières porcs et volailles, de la traçabilité des lots d'animaux, a obligé l'éleveur à enregistrer et à conserver quotidiennement tous les mouvements dans son atelier. Ceci se traduit, au sein des Bilans Travail réalisés, par une tâche administrative régulière qu'il ne faut pas différer et donc assimilée à du travail d'astreinte. De plus, l'obligation de conserver une trace écrite de certains documents sur une durée comprise entre 3 à 5 ans incite fortement les éleveurs à créer un bureau, permettant aussi de mieux séparer la partie professionnelle de la partie privée.

\section{3 / Gestion des risques sur la santé des éleveurs}

L'évolution des techniques et des technologies a permis de diminuer la charge physique que doivent supporter les éleveurs, mais ce n'est pas le seul risque auxquels les éleveurs doivent faire face. Ainsi, au cours des vingt dernières années, la dimension santé et sécurité au travail a été une composante importante des études réalisées sur le thème du travail en aviculture. L'élevage avicole ou porcin ne présente 
pas plus de risques que d'autres activités d'élevage, il en présenterait même plutôt moins eu égard à la taille des animaux élevés. Mais il expose à des risques spécifiques, liés notamment à l'élevage en bâtiments le plus souvent fermés, au type de tâches à réaliser ou à la nature des animaux côtoyés, qui peuvent avoir un impact sur la santé des éleveurs. Les quantités importantes d'animaux, de nourriture et de déjections, ainsi que les gaz résultant du chauffage des bâtiments, sont sources d'émissions de poussières, d'ammoniac, de monoxyde de carbone, de germes bactériens ou viraux en suspension potentiellement toxiques pour l'arbre respiratoire des éleveurs (Donham 1990). D'autant que les activités, rythmées par la conduite en bandes des troupeaux, nécessitent, au moins à certaines étapes du cycle de production, une présence permanente et prolongée dans ces bâtiments. Au final, les pathologies d'origine professionnelle observées dans le secteur avicole sont de quatre ordres (Abadia et Mirabito 2003) : i) pathologies respiratoires, ii) pathologies cutanées, iii) pathologies d'origine physique dues à des hyper-sollicitations des articulations (troubles musculo-squelettiques) et $i v$ ) pathologies infectieuses et zoonoses, liées à l'élevage (notamment infection par Chlamydophila psittaci en élevage de canard) ou par injections accidentelles au cours des opérations de vaccinations.

Le risque de pathologies respiratoires est substantiel. Les aviculteurs (et les porchers) montrent des signes d'altérations, plus ou moins prononcés, des fonctions respiratoires (Gérault et al 2003) par rapport à une population de référence composée d'agriculteurs et de salariés agricoles hors secteur monogastrique. Les produits désinfectants (à base de formol), les plumes des animaux et les poussières (alimentation en sec...) sont des facteurs qui augmentent potentiellement les risques et pourraient être à l'origine de ces symptômes.

Les pathologies d'hyper-sollicitation apparaissent sous l'effet de facteurs prédisposant d'ordre professionnel (gestes répétés, cadences soutenues, position articulaire extrême), environnemental (froid) ou personnel (activités extraprofessionnelles sollicitant les mêmes articulations, stress et sans doute un effet sexe, avec une fréquence des pathologies d'hyper-sollicitation plus élevée chez les femmes). Les activités professionnelles exposant les aviculteurs à un risque d'hyper-sollicitation articulaire sont nombreuses et courantes (Abadia et Mirabito 2003): l'insémination, le débecquage, les vaccinations, le ramassage des volailles d'autant plus pénible que le poids de la volaille est important et que la durée de l'activité est limitée dans le temps. Sur le plan des infections, il existe plusieurs types d'affections susceptibles d'être transmises à l'homme par les oiseaux mais elles restent heureusement rares et peu transmissibles (chlamydioses aviaires, tuberculose à Mycobacterium avium, influenza aviaire...). Il ne faut pas négliger non plus les maladies non spécifiques aux oiseaux comme le tétanos ou les pathologies dues aux nuisibles et notamment aux rongeurs (leptospirose), également rares et contrôlables par une prévention efficace.

Tous ces risques sur la santé sont de mieux en mieux pris en compte dans les élevages notamment par la mise en œuvre d'innovation techniques (ventilation, ramassage automatique des œufs, trempage automatique lors du nettoyage des salles ou bâtiments) et par la réalisation de bâtiments fonctionnels (zone d'isolement, circulation des animaux...).

\section{4 / Vers une gestion collective et une gestion de collectifs}

Une des évolutions majeures de la dernière décennie est la place croissante d'une gestion collective dans les élevages. Ainsi, entre 2000 et 2008, le nombre de salariés dans les élevages bretons de «gros animaux» (bovins et porcs) est passé de 2682 à 3071 (OESR MSA, cité par Depoudent et Le Moan 2010). Gérer un salarié demande de mettre en œuvre des compétences en management et gestion des relations humaines (Le Moan et al 2007). Ce passage du statut «d'éleveur» au statut «d'employeur»a généralement lieu dans de bonnes conditions. Toutefois, une bonne anticipation des connaissances et compétences à acquérir est nécessaire.

L'augmentation de la taille des élevages n'est pas le seul facteur générant une gestion plus collective de certaines tâches. Dans le cadre de la mise aux normes sur le bien-être, certains éleveurs remplacent leur activité de naissage par la participation à une maternité collective. La gestion des relations entre associés et avec les salariés se substitue alors à des tâches techniques gérées de manière plus indépendante (Grannec et al 2011). Dans une moindre mesure, l'obligation de traiter les effluents dans le cadre de la réglementation sur le phosphore génèrera vraisemblablement un développement de la gestion collective d'outils de traitement (Grannec et Chevance 2011).

Enfin, la réduction de la part de la population ayant des origines agricoles demande de renouveler les logiques de communication entre les agriculteurs et les autres utilisateurs de l'espace rural.
Expliquer, communiquer, mais surtout construire collectivement des règles de fonctionnement sont indispensables à la pérennité des élevages (Grannec 2010b).

\section{4 / Des évolutions de systèmes qui font et feront réfléchir sur le travail et son organisation}

Quelles évolutions attendre pour les années à venir sur le travail dans les filières monogastriques ? Les systèmes paraissent déjà très optimisés et très automatisés. Il reste des marges de progression, tous les élevages n'étant pas au même niveau d'optimisation. De fait, les conditions de travail moyennes de l'ensemble des élevages devraient progresser. Mais certaines tendances d'évolution en élevage porcin (truies en groupe, agrandissement...) et avicole (passage vers des cages aménagées ou vers de l'élevage au sol, agrandissement) vont avoir des conséquences sur le travail des éleveurs qui sont peu souvent estimées. Dans ce domaine, la filière avicole semble avoir pris de l'avance en ayant innové pour répondre à des soucis de travail des éleveurs (dans les filières dindes de chair et poules pondeuses label et biologique que nous développons ci-dessous) ou en analysant les conséquences en terme de travail des nouvelles réglementations (filière palmipèdes gras).

La filière dinde de chair utilisait avant 2009, une souche, véritable «formule un» au niveau des performances mais qui était devenue trop fragile en élevage et engendrait un surcroît de travail pour les éleveurs (paillage du bâtiment tous les 2 jours) et de stress tout au long du lot, à tel point que certains éleveurs souhaitaient arrêter cette production. De plus, le temps passé à veiller au déroulement du lot ne se traduisait pas forcément de manière économique. L'arrivée de deux nouvelles souches, plus robustes fut une véritable bouffée d'oxygène. Le retour à des performances techniques et économiques plus qu'honorables avec une charge de travail régulière permit de renouer avec l'envie de continuer à produire de la dinde de chair standard.

Dans le même temps, en poules pondeuses label et biologique, une nouvelle souche plus performante fit son apparition. Celle-ci pouvait pondre plus d'œufs mais avait tendance à pondre non pas dans les nids dédiés à cet effet mais au sol, entraînant une surcharge de temps et une pénibilité du travail. La stratégie pour pallier ce souci fut de mettre en place une phase de «dressage» 
pour éduquer la poule durant toute la période de montée en ponte de la jeune poulette. Cette phase se caractérise par de nombreux passages au milieu des poules afin qu'elles ne restent pas au même endroit et qu'elles recherchent le calme des nids pour y pondre (Morinière et Pineau 2010). Le temps d'astreinte, durant cette période, s'est trouvé augmenté d'une heure en moyenne par rapport à ce qu'il était avec l'ancienne souche.

La filière palmipèdes gras offre une autre illustration de changement de système de production ayant une incidence sur le travail du gaveur. Le remplacement de la cage de gavage individuelle par le logement collectif, à des fins de bien-être animal, s'il permet aux animaux d'exprimer un répertoire comportemental plus diversifié, engendre une manipulation plus délicate des canards qui peut être source de pénibilité (Mirabito et Sazy 2004). L'opérateur doit se baisser et se pencher, avec une mise en tension du bas du dos. La force à exercer par le gaveur, en l'absence d'un système de contention, est aussi plus importante. Le changement de système génère de nouvelles contraintes, mais il peut aussi en alléger d'autres. Une enquête, réalisée auprès de gaveurs ayant modifié leur système d'élevage ces dernières années (Blériot 2004), montre que les parties du corps signalées comme les plus douloureuses sont les doigts et les mains pour les gaveurs avec cage individuelle et alimentation en maïs broyé (en relation sans doute avec les fortes cadences permises par certains types de gaveuses), tandis que les parcs traditionnels semblent générer des douleurs plus fréquentes au niveau dorsal. Le mode de logement n'est sans doute pas le seul critère à prendre en considération : le type d'alimentation, le type de gaveuse et le comportement des canards ont aussi un effet sur les conditions de travail, montrant toute la palette des facteurs sur lesquels il est possible d'intervenir pour modifier les conditions de travail, et de fait toute la complexité d'une analyse globale.

Pour les deux filières, l'agrandissement des structures d'élevage tend à s'accompagner du développement d'élevages «multisites» afin de répartir les zones de production d'effluents notamment. Cette structuration pose des questions pour l'organisation du travail (affectation du personnel par site, spécialisation des sites, alternance des pics de travail entre les sites...) et chaque éleveur devra trouver sa propre solution pour mettre en œuvre son système d'élevage (embauche d'un salarié, automatisation...).

\section{Conclusion}

Au final, le temps et l'organisation du travail dans les élevages porcins et avicoles sont très variables selon les systèmes d'élevages, la place que donne l'éleveur à ses ateliers, la mécanisation des tâches etc. Le métier en lui-même est aussi en pleine évolution en raison de l'augmentation de la part du salariat dans les exploitations et de la présence de l'automatisation. Ainsi, toutes les modifications de système qui sont en cours dans le cadre des mises aux normes vont entraîner des modifications dans le travail des éleveurs (gestions collectives, augmentation de la durée de certaines tâches). Ces modifications des différentes dimensions du travail devraient être mieux prises en compte dans les activités de conseil et de recherche car c'est aussi dans ces dimensions sociologiques que se trouvent l'attractivité du métier et l'acceptabilité de solutions techniques (Fiorelli et al 2010).

\section{Références}

Abadia G., Mirabito L., 2003. Influence des modifications des systèmes d'élevage sur la

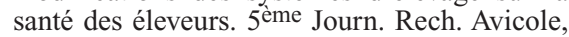
Tours, France, 41-48.

Agreste, 2008. Recensement agricole et enquêtes sur les structures d'élevage du Service de la Statistique et de la Prospective (SSP) du Ministère de l'Agriculture, http://www.agreste.agriculture.gouv.fr/enquetes/structure-des-exploitations/

Bartolomeu D., 2005. Les temps de travaux en élevage, points clés et facteurs de variation. Techni Porc, 28, 3-12.

Bassez J., 1973. Le temps de travail en porcherie. Tribune du monde rural, $2 \mathrm{p}$.

Bassez J., 1976a. En élevage, la prévision du travail journalier est possible : application à l'élevage des truies en bande. B.C.M.E.A., Informations équipement, 5, 46-50.

Bassez J., 1976b. En élevage, la prévision du travail journalier est possible : application à l'élevage des truies en bande. Deuxième partie : exemple de calcul d'un planning de travail pour un atelier naisseur-engraisseur, de 80 90 truies, conduit en bande. B.C.M.E.A., Informations équipement, 6, 16-23.

Blériot G., 2004. Rapport de fin d'étude, Ecole Nationale Supérieure d'Agriculture de Rennes-ITAVI.

Caugant A., 2002. La conduite en bandes en élevage porcin. EDE Bretagne, Chambres d'Agriculture de Bretagne, 28p.

Chevalier D., Guerder F., 2004. Temps de travaux en élevage de multiplication. Journée
Nationale des élevages de multiplication et d'accouvage. Rennes, France, 39p.

Commandeur M., Le Guen R., Dourmad J.Y., Casabianca F., 2006. La diversité des styles d'élevages porcins : une approche dans les Côtes d'Armor. Journ. Rech. Porcine, 38, 247254.

Corrégé I., Theil G., 2008. Facteurs d'optimisation du protocole de nettoyagedésinfection en élevage : importance des modalités de trempage. Journ. Rech. Porcine, $40,51-52$.

Daelemans.J., 1972. Les besoins en maind'œuvre dans l'élevage porcin. Traduit du néerlandais. Revue de l'Agriculture, 25, 1083-1091.

Depoudent C., Le Moan L., 2010. Fidéliser et motiver une équipe de salariés. Chambres d'Agriculture de Bretagne, 46p.

Depoudent C., Grannec M.L., 2011. Spécificités du salariat féminin en élevage porcin. Journ. Rech. Porcine, 43, 253-254.

Depoudent C., Le Moan L., 2011. Déterminants de la fidélisation d'une équipe de salariés en élevage porcin. Journ. Rech. Porcine, 43, 255-256.

Depoudent C., Grannec M.L., Quillien J.P., 2012. Représentations et réalités du salariat féminin en élevage porcin. Journ. Rech. Porcine, 44, 245-246.

Dockès A.C., Kling-Eveillard F., 2007. Les représentations de l'animal et du bien-être animal par les éleveurs français. In : Numéro spécial, Bien-être animal. INRA Prod. Anim., 20, 23-28.
Donham K., 1990. Health effects from work in swine confinement buildings. Am. J. Ind. Med. 17, 17-25.

Fiorelli C., Dedieu B., Porcher J., 2010. Proposition d'un cadre d'analyse des compromis réalisés par les éleveurs pluriactifs pour organiser leur travail. Cah. Agric., 19, 383-390.

Gallot S., 2006. Caractérisation des exploitations avicoles françaises à partir de l'enquête aviculture 2004. ITAVI. 186p.

Gallot S., 2008. Le travail en aviculture. Institut de l'Elevage / ITAVI, 15p. http://www.inst-elevage.asso.fr/spip.php?article19153

Gallot S., 2011. Référentiel temps de travail : synthèse détaillée des filières avicoles. Editions Institut de 1'Elevage, Paris, France, 20p.

Gérault P., Dewitte J.D., Jourdren L., 2003. Affections respiratoires des aviculteurs et des porchers. Journ. Rech. Avicole, Tours, France, $4 \mathrm{p}$.

Grannec M.L., 2010a. Référentiel travail synthèse de 23 bilans travail, Institut de l'Elevage/Chambre Régionale d'Agriculture de Bretagne, 19p, http://www.instelevage.asso.fr/spip.php?article18977

Grannec M.L., 2010b. Pour Yvon et Bernadette Gicquel, agrandissement d'exploitation rime avec communication. Atout Porc Bretagne, 58, 34-35.

Grannec M.L., Chevance C., 2011. naisseurs-engraisseurs de moins de 150 truies en Bretagne. Journ. Rech. Porcine, 43, 223-227. 
Grannec M.L., Bordes A., Pellois H., Boulestreau-Boulay A.L., Grimaud P., Pineau P., 2011. Maternités collectives : une stratégie d'avenir pour les naisseurs-engraisseurs ? Journ. Rech. Porcine, 43, 249-250.

Guerder F., 2001. Les temps de travaux en aviculture : enquêtes en élevage de poulets, poulets label, dindes, canards et pintades. Rapport OFIVAL. 30p.

Ilari E., Chatellier V., Marouby H., 2007. Les exploitations agricoles avec porcins en France face à la réforme de la PAC. Journ. Rech. Porcine, 39, 167-172.

I.T.P., I.G.E.R., 1973. Temps de travail dans les ateliers de production porcine. Rapport d'étude, Ministère de l'Agriculture, 142 p.

Jégou J.Y., Larour G., Pellois H., Ramonet Y., 2010. Les conduites en bandes en production porcine, $44 \mathrm{p}$.

Le Borgne M., 1992. La productivité du travail en atelier naisseur-engraisseur porc. EDE 22. Revue du groupement Porcial.

Le Borgne M., Quentric O., 2002. Temps de travail : une meilleure efficacité. Atout Porc Bretagne, 30-32.

Le Borgne M., Le Bris B., Quillien J.P., 1994. Temps de travaux : enquête dans 89 ateliers porcins de Bretagne. EDE-Chambres d'Agriculture de Bretagne.

Le Moan L., Pichodo X., Roy H., Quinio P.Y., Renault E., Le Borgne M., Le Cozler Y., Pellois H., Bartholomeu D., Donet P., Sallard R., 2003. Temps de travail en élevage porcine, Bretagne, Pays de la Loire. Chambres
d'Agriculture de Bretagne, Programme Porcherie Verte, Action Ac-11a, 41p.

Le Moan L., Pineau C., Grimaud P., 2007. Conséquences techniques, économiques et sociales de l'embauche du premier salarié en élevage porcin. Chambres d'Agriculture de Bretagne, 84p.

Le Moan L., Larour G., Pellois H., 2009. Conduites en bandes, partie 3 - organisation du travail et conduites en bandes. Chambres d'Agriculture de Bretagne, 78p.

Martel G., Dourmad J.Y., Dedieu B., 2008. Do labour productivity and preferences about work load distribution affect reproduction management and performance in pig farms? Livest. Sci., 116, 96-107.

Mirabito L., Sazy E., 2004. Le logement collectif : vers un compromis entre le bien-être des canards et le confort des éleveurs ? Journ. Rech. sur les Palmipèdes à Foie Gras. Arcachon, France.

Morinière F., Pineau C., 2010. Le travail en élevage de poules pondeuses biologiques. Réseau Bio des Chambres d'Agriculture des Pays de la Loire, 6p.

Nicourt C., Souron O., 1989. Incidence de quelques innovations sur les conditions de travail des agriculteurs. Economie Rurale, 192193, 110-114

Pineau C., Morinière F., 2009. Bien conduire son lot de poules. Dans «Produire des œufs biologiques», Cah. Tech. de l'ITAB, 10-12.

Ramonet Y., Chopin E., Caille M.E., Dubois A., 2010. Les truies gestantes en groupes au
Dac - enquêtes en élevages et auprès d'équipementiers. Chambres d'Agriculture de Bretagne, 40p.

Reboul C., 1964. Temps de travaux et jours disponibles en agriculture. In : Économie rurale. Milhau J., Montagne R. (Eds). 61, 55-80.

Renaud H., 2010. Le temps de travail en élevage porcin : facteurs de variation et voies d'amélioration. Mémoire d'Ingénieur, Ecole d'ingénieurs de Purpan, $89^{\mathrm{e}}$ promotion, 66p.

Riffard C., Gallot S., 2010. Caractérisation des exploitations avicoles françaises à partir de l'enquête aviculture 2008. ITAVI. 161p.

Roguet C., 2011. Situation économique et financière des exploitations porcines dans cinq pays de l'UE de 2004 à 2008. Techni Porc, 34, 3-8.

Roguet C., Rieu M. 2011. Essor et mutation de la production porcine dans le bassin nordeuropéen : émergence d'un modèle d'élevage transfrontalier inédit. Notes et Etudes SocioEconomiques, 35.

Roguet C., Renaud H., Duflot B., 2011. Productivité du travail en élevage porcin : comparaison européenne et facteurs de variation. Journ. Rech. Porcine, 43, 251-252.

Salaün Y., 1987. Une approche du temps de travail en production porcine à partir des résultats des élevages du réseau dit «tableau de bord I.T.P.». Techni Porc, 10, 73-83.

Salaün Y., 1992. Analyse du temps de travail dans les élevages suivis par le «tableau de Bord ITP» en 1991. Techni Porc, 15, 4-92.

\section{Résumé}

Les élevages de porcs et de volailles sont souvent perçus comme «industriels» de fait du mode d'élevage en bâtiment et des rythmes réguliers de production. Cette perception entraîne l'image d'une organisation et d'une durée du travail très réglées et homogènes entre les élevages. Or, les études menées dans ces filières montrent une grande diversité d'attentes, de stratégies, de durée et d'efficacité du travail, en lien avec les évolutions structurelles du secteur. Ces évolutions sont différentes entre les deux filières : en production porcine, on observe une augmentation de la taille des ateliers, un arrêt du naissage pour les petites structures, la création de naissages collectifs et plus de salariat ; en production avicole, la concentration est modérée, liée au fort développement des productions alternatives et à l'absence de spécialisation des exploitations avicoles. En production porcine ces évolutions sont nuancées par l'analyse des structures européennes. D'un point de vue économique, toutes les stratégies peuvent être efficaces, les unes en réduisant les charges, les autres en maximisant la production par travailleur. A l'échelle des exploitations, il existe dans les deux filières une forte variabilité des temps de travaux entre exploitations, résultant principalement du type de bâtiment et d'équipement choisi, de la mécanisation, du type d'élevage, du nombre de bandes choisies, mais aussi de la place que donne l'éleveur à son atelier. Pour l'organisation du travail intra- et inter-hebdomadaire en production porcine, les différences résultent des choix de conduite en bandes et des pratiques de reproduction. Enfin le contenu du travail est en évolution : développement de compétences managériales, réduction des tâches exigeantes (via l'automatisation) remplacées par des travaux de surveillance, de gestion de l'information et de suivi de traçabilité. Pour conclure nous illustrons l'effet du changement d'un élément du système d'élevage sur le contenu, la durée, l'organisation ou la pénibilité du travail.

\section{Abstract}

\section{The work of pig and poultry farmers: a large diversity of strategies, expectations, durations and productivity}

Pig and poultry productions are often perceived as "industrial" since animals are kept indoors and the production rhythms are regular. This perception leads to the image of a work organization and duration that follows a similar schedule being consistent between farms. However, studies on the labor in those productions show a wide range of expectations, strategies, durations and work efficiency in relation with structural changes of the sector. In pig production, farm size increases, the use of hired labor also, and small farms disappear, in particular those with sows. In poultry production, the size increase is moderate, the alternative productions are common and farms are less specialized. Those evolutions are compared to European pig production. From an economical point of view all the strategies can be effective, the one reducing the charges, the other maximizing output per worker. At the farm scale, the stu-dies show a large variability of working time between farms in the two type of productions, which is mainly due to facilities and 
equipment, mechanization, the kind of species bred, batch number but also the role given by the farmer to the unit. Inter- and intraweek work organizations in pig production differ with the batch farrowing system and breeding practices. Finally, job content is changing: development of managerial skills, reduction of exhaustive tasks replaced by supervising tasks, data management and traceability monitoring. To conclude we illustrate the effect of a change in the production system on content, duration, organization and tiredness of the farmer's work.

MARTEL G., DEPOUDENT C., ROGUET C., GALLOT S., PINEAU C., 2012. Le travail des éleveurs avicoles et porcins : une grande diversité de stratégies, d'attentes, de durée et de productivité. In : Numéro spécial, Travail en élevage. Hostiou N., Dedieu B., Baumont R. (Eds). INRA Prod. Anim., 25, 113-126. 